\title{
Play, Pause, and Skip: touch screen media player interaction on the move
}

\author{
Nicholas Vanderschantz ${ }^{[0000-0002-7729-7936]}$ and Zhiqin Yang \\ Computer Science Department, University of Waikato, Hamilton, New Zealand

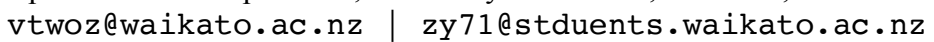

\begin{abstract}
Our active lifestyles see us playing, pausing, and skipping through life all the while our phones are in our hands. For many, completing the daily grind requires regular audio and visual media accompaniment and for this we interact with our phones as we skip, run, and jump. In this respect, a unique form of digital library is our mobile media player of choice. These media players serve as both the interface for listening to and watching these audio and visual media as well as the media library and storage. We argue therefore that the interface design considerations of the media library as well as the media interaction require user centered investigation. We tested button placement variations and analyzed the user preferences as well as user interaction with these mobile media player prototypes while on the move. Early insights suggest users prefer what they are most accustomed to, yet issues of accuracy with interface designs that are unfamiliar require further investigation.
\end{abstract}

Keywords: User Interaction, Touch Screen, Media Player, Mobile Design.

\section{$1 \quad$ Introduction}

There are few parts of society that the smartphone is not found, and our daily commute and regular exercise are likely no exception. When on the move we are often found tapping, scrolling, texting, checking email, and listening to our favorite tune. This common use of mobile phones for music playback is undeniable, with evidence of substantial decrease of dedicated portable media player sales for some time now [7]. Punchoojit \& Hongwarittorrn [12] recently completed a broad survey of usability studies of mobile user interface design and have concluded that further empirical evidence is still required to guide designers and developers in creating efficient and effective interactive touch screen interfaces for use while users are moving. We present one of the few studies into mobile use of media player interfaces on touchscreen devices and show that there is need for further investigation into button placement for users in motion. 


\section{Related Work}

Investigations of touch screen interaction has explored a range of devices and screen sizes as well as sizing, spacing, and placement of GUI features. Few investigations have considered the impact of interface design on device use during movement [i.e., 3]. Of the recent digital library interface design research for mobile touch screen use, the majority of this work has focused on user preferences regarding the display of information [i.e., 16] rather than user interaction with particular media. We focus the discussion of the related work on research relating to design and implementation of interactive touch screen features of smartphone applications.

\subsection{Button Size \& Spacing}

Generally accepted target size recommendations for single click buttons range from $8 \mathrm{~mm}$ to $14 \mathrm{~mm}$. Parhi et al., [10] found that touch target sizes larger than $9.6 \mathrm{~mm}$ showed the lowest error rates. Park \& Han [11] studied one-handed thumb interaction concluding that a $10 \mathrm{~mm}$ wide button provided the best usability. Tao et al., [14] found that users preferred and were more efficient using $17.5 \mathrm{~mm}$ square buttons during text and numeric input.

Of the few investigations of mobile interface use during movement, Conradi et al., [3] investigated optimal button sizes for use while users are walking. Users were able to use the $8 \mathrm{~mm}$ sized buttons, but they made more mistakes than with $14 \mathrm{~mm}$ buttons. This relates to the findings of Chourasia et al., [5] who found that users made more mistakes during number entry using smaller buttons when standing than when sitting.

Unsurprisingly when the spacing of buttons is larger user interaction times are increased. Jin et al., [6] investigated button size and padding on tablet sized touch screens with consideration of reaction time and accuracy and recommend button spacing of between $3.17 \mathrm{~mm}$ and $12.7 \mathrm{~mm}$. Similarly, Sun et al., [13] found that their users preferred a spacing of $6.35 \mathrm{~mm}$ to $12.7 \mathrm{~mm}$.

\subsection{Button Location}

Much of the button location research focusses on supporting users holding and using a device with the same hand. Park \& Han [11] proposed a way to analyse and describe location of elements on a mobile screen by dividing the device screen into twenty-five areas. When a user held the device with their right hand and interacted with their thumb, Park \& Han recommended placing interactive items in the centre of a touch screen because these locations were faster to interact with and preferred by users of their study. Buschek [2] also investigated thumb interaction, and tested prototype UI interaction methods for common mobile GUIs. From their UI experiments they recommend that designers reconsider default locations and presentations of typical GUI features. Karlson et al., [9] recommended placing points of interaction centrally to accommodate both left- and right-handed users. 


\subsection{Holding and Using a Device}

Hoober [8] showed that only $15.8 \%$ of the users used their left hand to touch the screen. Cornelia [4] divided her results into when participants were sitting, standing or walking and confirmed that most of the participants used their right hand to touch the screen irrespective of what they were doing at the time. Users are known to change their hold for certain interactions and research shows some hand holds are more successful than others for text entry on mobile touchscreen devices [1]. Reinforcing the importance of button layout and location on a touchscreen device Trudeau et al., [15] showed that different button positions require users to adjust the way that they hold their device.

\section{Case Study}

We conducted a case study audit of the typical design and placement of play/pause, last track and next track buttons in iOS and Android music player apps. To identify appropriate music player apps to study a Google search ("most popular music player applications") was performed. Apps common to both device platform as featured in the results on the first page of this Google search were selected. For this case study we reviewed Spotify, SoundCloud, YouTube Music, and Google Play Music, and excluded apps solely for music discovery, karaoke, or available only on a single platform.

All apps were audited on an iOS iPhone 6s and an Android Nexus E960 which have similar physical dimensions.

\subsection{Case Study Results}

Given the limits of this paper and the nature of this explorative case study we report a summary of our findings which were used to guide the development of our user study.

Button Design: Button iconography for media players has roots in physical media player buttons and therefore follows expected traditions of the highly recognised icons of a single right facing triangle for play buttons, two vertical bars for pause buttons, and either two interlocked triangles or a triangle and vertical bar for last track and next track buttons. For all of the apps reviewed, only play/pause icons were encapsulated in a shape - always a circle.

Button Size: Only the identifiable touch area or containment shape, or the icon itself was measured. The most typical size of play and pause buttons with a circle were $8.8 \mathrm{~mm}$ to $13 \mathrm{~mm}$ and $3.9 \mathrm{~mm}$ to $6.8 \mathrm{~mm}$ buttons without. Last and next track buttons were never contained within a shape and the icon sizes measured between $2 \mathrm{~mm}$ to $4 \mathrm{~mm}$.

Buttons Layout and Spacing: In all instances the play/pause button was placed in the middle of the three buttons with the last track button on the left and the next track button on the right - a horizontal layout. Button spacing was always consistent between these three buttons and ranged from $4.5 \mathrm{~mm}$ to $22.6 \mathrm{~mm}$ between the visible edges.

Button Location: To ascertain commonalities of button location we took guidance from Park \& Hans [11] method of dividing the device screen into twenty-five areas. The findings indicate that interface areas were most likely to be placed at approximately the vertical-centre of the screen or below. 
A typical example of the button design, size, layout, and spacing that we observed in this case study is portrayed in Figure 1.

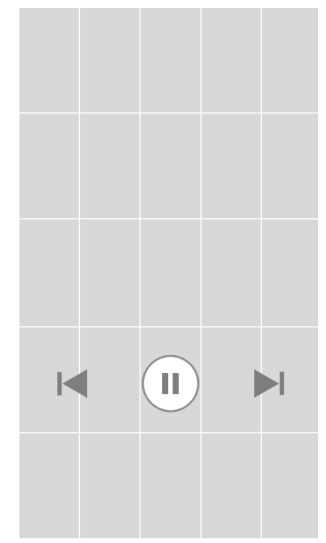

Fig. 1. Typical button design, sizing, and layout for the four interfaces reviewed across the two operating systems (eight interfaces total reviewed)

A representation of the button locations of the eight interfaces reviewed is provided in Figure 2. We use the Park \& Han [11] method of representing a screen as 25 divisions to assist with this visualisation.

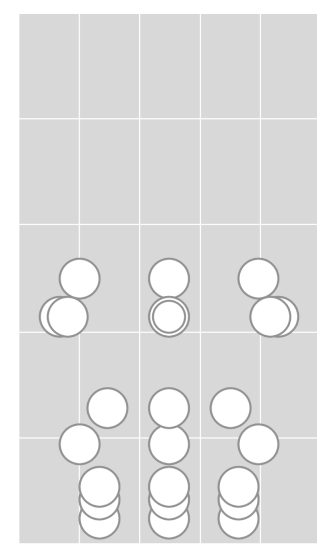

Fig. 2. Representation of observed button locations for the four interfaces reviewed across the two operating systems (eight interfaces total reviewed)

\section{User Observation Study}

We followed our case study with a user observation study of 30 participants interacting with six media player interface prototypes while walking. We conducted an observed interaction study with in-situ interview questions after the use of each test condition. 


\subsection{User Study Method}

We designed and implemented six media-player interface prototypes using Proto.io (Proto.io, 2018). The prototypes were explored by 15 female and 15 male participants using an iPhone 6s. Ethical approval was received by the university to conduct this research and all participants consented to participation before the study was conducted. Participants followed verbal instructions for the use of the six prototype test conditions. Field notes and audio recordings were taken during the observation and interview.

\section{User Study Test Materials.}

We used our findings from the related work (see Section 2) and our own case study (see Section 3) to assist with the design and implementation of six media-player interface prototypes. This section describes the design considerations used when developing the six test conditions tested in this study.

The location and orientation of buttons are the two variables that were of interest for this study. For this reason, we controlled other interface variables with all test conditions using the same button designs, sizes, and spacings. We tested three horizontal conditions and three vertical conditions (see Table 1). This allowed for testing preferences and successful use of elements in horizontal and vertical layout of interactive elements.

Table 1. Button locations in six test conditions

\begin{tabular}{|l|l|l|}
\hline Label & Test Condition & Screen Location, Button Orientation \\
\hline TC1 & Test Condition 1 & Centre location, horizontal orientation \\
\hline TC2 & Test Condition 2 & Middle bottom location, horizontal orientation \\
\hline TC3 & Test Condition 3 & Left bottom location, horizontal orientation \\
\hline TC4 & Test Condition 4 & Centre location, vertical orientation \\
\hline TC5 & Test Condition 5 & Middle bottom location, vertical orientation \\
\hline TC6 & Test Condition 6 & Left bottom location, vertical orientation \\
\hline
\end{tabular}

In line with recommendations and observations we designed and implemented play, pause, last track and next track buttons using typical iconography to ensure visibility and recognition. We implemented buttons that had an $11 \mathrm{~mm}$ diameter circle denoting the interactive tap space for each of the buttons. Due to the use of a containment shape for our buttons and in-line with recommendations we have spaced the buttons $3.5 \mathrm{~mm}$ from edge to edge of the adjacent button. This resulted in buttons with a center-tocenter spacing of $14.5 \mathrm{~mm}$.

Our interface test materials can be found in Appendix A.

\section{User Study Procedure.}

Observations: sessions begun with the participant and researcher sat at a table. Following discussion of the procedure and participant consent, the researcher asked the participant to pick up a phone placed in front of them. The participants were then asked 
to stand and walk along a foyer and follow verbal instructions for use of the prototype given by the researcher. Each participant used all six interface conditions (see Table 1 \& Appendix A). To avoid effects of order-bias, we divided the 30 users across six intervention orders.

The participants were observed by the researcher as they interacted with the prototype on the phone. The researcher sat with the user during the initial phase of the observation, and stood and walked with the user during the movement phase of the experiment. If the participants successfully tapped a button, the interface presented a success page, while inaccuracy resulted in the user needing to tap again.

Interviews: after interacting with an interface, the user was asked an open-ended perception question regarding ease of use. Before being presented with the next test condition, each user was also asked to rank the interface on a 5-point Likert Scale from very easy to very hard. Following the use of all six test conditions the users were also asked which condition was most and least comfortable to use.

\subsection{User Observation Study Results}

Here we report the results of our study with 30 participants aged 20 to 60 years old. While we had four participants who were over the age of 30 , the remaining participants were aged between 20 and 29 years old and therefore we do not attempt to infer age related statistical insights.

\section{Device Pickup \& Hand Switching.}

Of the 30 participants, 20 picked up the device with their right hand, two picked up the device with their left hand. The remaining eight participants ( 5 female, 3 male) picked up the device with both hands.

Six participants switched their hands when they were instructed to click the first button in the first test condition they were given. Three participants switched hands in order to tap the first requested button on $\mathrm{TC} 1$, (center location, horizontal orientation). For each of TC6 (left bottom location, vertical orientation), TC5 (middle bottom location, vertical orientation) and TC2 (middle bottom location, horizontal orientation), one participant switched hands in order to tap the first button.

\section{Interaction.}

When interacting with the prototypes the 30 participants, tapped three buttons in each test condition, therefore each test condition received a possible 90 interactions, and thus we observed a total of 540 interactions.

414 taps were made by a right thumb or right finger compared to 126 made by a left thumb or finger. 372 single-handed interactions were made compared to 168 twohanded interactions. Overall use of two-handed operation was higher for horizontal orientations (91 taps) than vertical orientations (77 taps). Similarly, total right-handed operation was higher for horizontal orientations (214 taps using a right thumb or right finger) compared to vertical orientations (200 taps). 
Hand Holds.

Four methods of holding the device while interacting were identified (see Table 2).

Table 2. Four methods of interacting with the device

\begin{tabular}{|l|l|l|}
\hline Label & Hand Hold & Description of Hold \\
\hline LH+T & Single-handed & Device in left hand tap using left-hand thumb \\
\hline RH+T & Single-handed & Device in right hand tap using right-hand thumb \\
\hline LH+RF & Two-handed & Device in left hand tap using right-hand finger \\
\hline RH+LF & Two-handed & Device in right hand tap using left-hand finger \\
\hline
\end{tabular}

The most used method of interacting with the prototypes was by holding the device in the right hand and using the right-hand thumb ( $\mathrm{RH}+\mathrm{T})$ a single-handed interaction. The second most used method was the two-handed interaction where the user held the device with the left hand and tapped using a finger on the right hand (LH+RF). The third most used method was the single-handed LH+T with very few participants using $\mathrm{RH}+\mathrm{LF}$. Figure 3 shows aggregated results of the method of interaction for each tap made by the users across each of the test conditions.

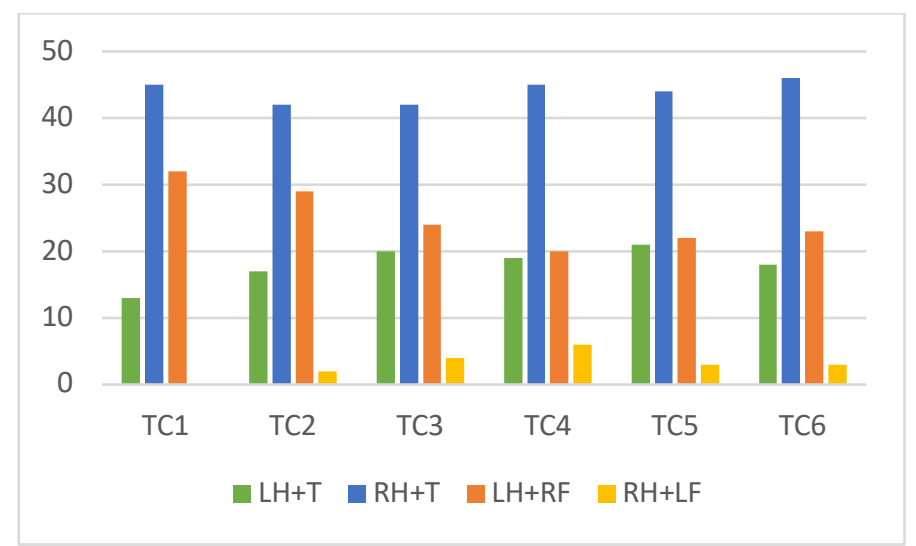

Fig. 3. Aggregated results of interaction method made by the users across each test condition

\section{Adjustment During Interaction.}

We report here further detail regarding users who adjusted their interaction method while using a particular test condition. Figure 4 shows the changes that occurred; for example, $\mathrm{P} 4$ used her left hand to tap both the play button and the next button on the horizontal TC1 but used her index finger on her right hand to touch the last track button which would have required her to bend her thumb uncomfortably. P4 also used her left hand and thumb to interact with the vertical TC6, swapped hands to use her right hand and right thumb for the next track button and then swapped back to her left hand and left thumb for the last track button. 


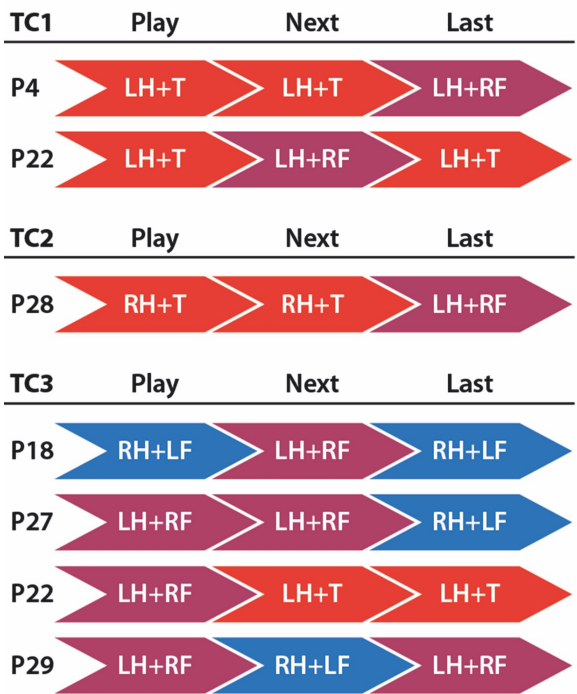

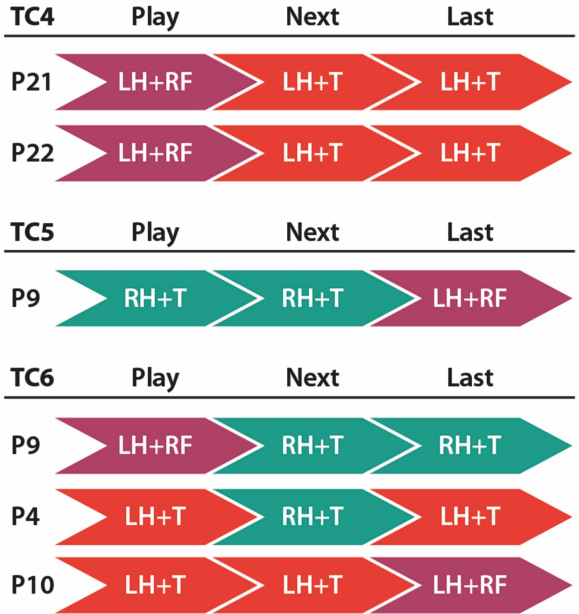

Fig. 4. Representation of the ways participants adjusted their hand holds during interaction

In total nine of 30 participants needed to adjust their hold during their interactions with one of the test conditions. Two of those nine participants adjusted their hand hold twice and one participant adjusted hand hold three times. Seven hand hold adjustments occurred when using a horizontal condition (see left-hand column of Figure 4), while six adjustments were needed while using a vertical condition (right-hand column of Figure 4).

\section{Accuracy.}

In our study, none of the participants tapped the wrong button and only seven of the participants did not successfully click their intended button the first time they attempted to. That is to say, that of the 540 instructions to tap, only seven inaccurate or unsuccessful attempts to tap were recorded. Inaccuracies were self-identified by participants who directly after a miss-tap, corrected their aim and tapped a second time on the target button they had missed with their first attempt.

We recorded three inaccuracies in horizontal test conditions and four inaccuracies in vertical test conditions. The buttons that caused issues for users were TC1 play and last track (1 inaccuracy each), TC3 play (1 inaccuracy) TC4 play (2 inaccuracies) and next track (1 inaccuracy), and TC6 next track (1 inaccuracy).

In the horizontal conditions the inaccuracies were for users holding with their lefthand. Two of these inaccuracies were for two handed operation, while one was for lefthand and left-thumb interaction. In the vertical conditions the inaccuracies were for single-handed operation - three right-hand and right-thumb interactions, and a single left-hand and left-thumb interaction. 


\section{Perceptions of Ease.}

After a participant finished interacting with a test condition we asked our two questions regarding preference. Our first question asked "Was the interface easy to use?" "Why?" More participants reported that the horizontal conditions were easy to use than reported the vertical conditions were easy to use (see Figure 5). However, in all cases less than half of the participants stated that a condition was not easy to use.

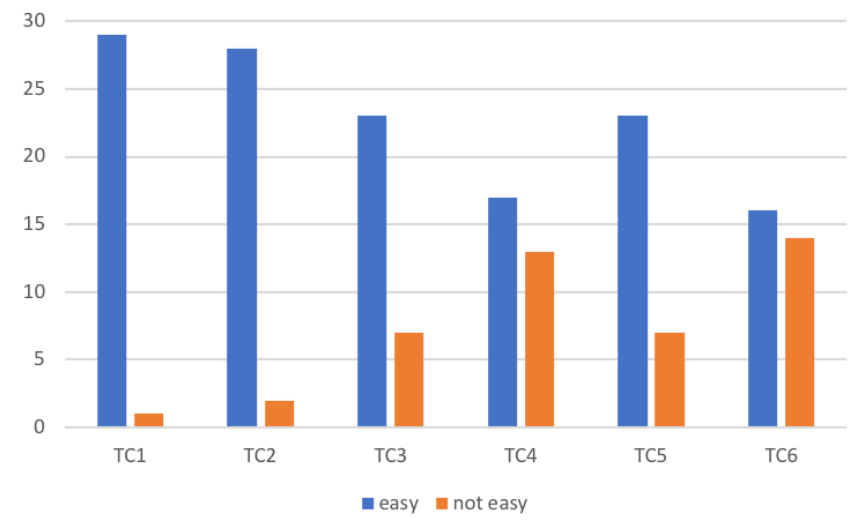

Fig. 5. Perception of ease for each interface

Our second question was a request for participants to rank the interface they had just worked with on a 5-point Likert scale from "very easy" to "very hard". Figure 6 shows that very few participants labelled any of the six test conditions as "very hard" or "hard". The test conditions that were thought to be hard included horizontal and vertical conditions, while the vertical conditions were the only conditions to be labeled as very hard when scored on the Likert scale. The numbers are very low in both instances.

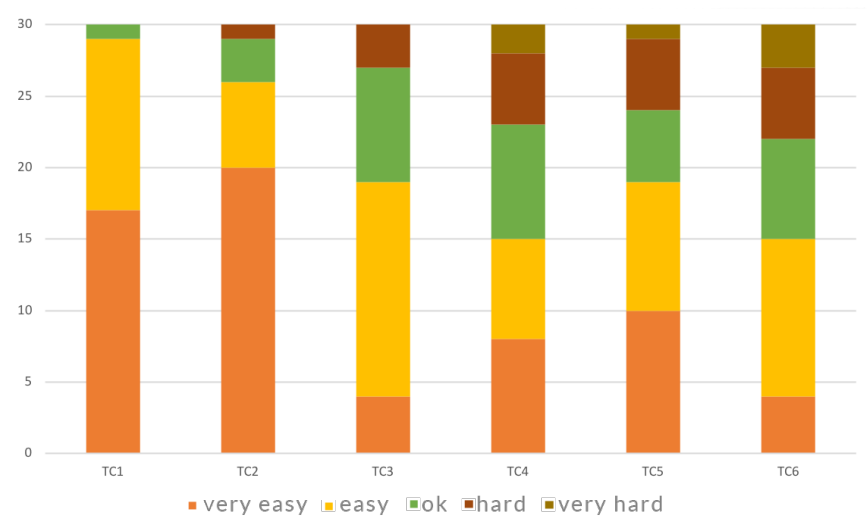

Fig. 6. Likert Scale perception of ease 
When we asked participants to qualify their answers, it was common for users to report that a TC was "easy to use", a button was "easy to reach", or they were familiar with a TC and this impacted their perception of ease. Similarly, common responses regarding why a TC was considered hard to use was because a button was "too far away to reach", or they were unfamiliar with a TC, or felt that aesthetically the interface looked "unusual", or "odd".

Reasons for both positive and negative responses were similar across the test conditions. For example, TC1 and TC4 both contain buttons located in horizontally and vertically central positions, and each received feedback regarding the central location of buttons being a positive feature.

Very few participants ranked vertical layouts as hard or very hard and this is reinforced in the qualitative feedback received. Vertical conditions often received feedback that suggested the participants were unsure about this layout. Participants reported that vertical conditions were unusual, and felt that they would require some getting used to. Participants used the word "odd" or "unusual" to describe this condition, and also noted for example "I'm not familiar with vertical placement but it is ok for using". One participant reported that they felt the vertical layout caused fewer mistakes for them when they were interacting with these interfaces.

\section{Discussion}

It is commonly held that interactive features on touch screen devices should fall in the lower portion and be centred horizontally in the screen [11,9]. Our analysis of popular music player interfaces, reveals that the most commonly used location for play/pause, last track, and next track buttons follows this belief.

Unique to our study was the inclusion of the non-typical vertical orientation of interface buttons. Vertical orientation resulted in higher left-handed operation and higher single-handed operation suggesting that further investigation is warranted. Confirming the findings of $[4,8]$, our user study showed the most common interaction in both a horizontal and vertical layout was right handed holding of the phone and interaction with the right thumb. The second most common interaction method in our study was a right finger interaction while the user held the phone with their left hand. This suggests that interfaces that consider right thumb or right index finger interaction will support the most users when they are moving.

Tao et al., [14] recommend considering both subjective and objective measures when developing touchscreen design guidelines. The subjective feedback regarding these vertical orientations was less positive than for the horizontal orientations. When questioned participants more often reported a preference for a horizontal button placement in touch screen mobile media player interfaces. This is almost certainly because the existing media player interfaces on mobile devices are typically arranged horizontally. It is therefore not unexpected that numerous participants reported that the vertical presentations were something they had little experience with and would require some getting used to. Given that very few participants ranked these vertical interfaces as very hard while other participants reported that the unusual interface would require further 
time to become comfortable may suggest that a study which allowed users more time to interact with the interfaces may alter the results of our study presented here.

\section{$5.1 \quad$ Limitations \& Future Work}

Our exploratory first study reported here sought to control a number of variables in order to identify potential interacting factors as well as opportunities for further investigation. With this in mind, this study controlled for location with all participants using the mobile device in the same physical location and beginning from the same seated position and phone placement. Future work would do well to consider varied environments such as indoor and outdoor use, navigation of entrances or exits to buildings, and inclining and declining terrain. Equally, it may prove interesting to have participants also place and remove the phone into their pocket, bag, or typical transport location as a feature of the study which may be an interaction similar to that of picking up the phone from the table for the first time. Placing the device back on the table may also prove insightful in understanding the ergonomics of common phone use.

A shortcoming of our method was that we did not ask users what their dominant hand was. This is an oversight when we find that total right-handed operation and twohanded interaction was higher in the horizontal orientations than the vertical orientations. These two results lead us to suggest that investigation of how orientation effects the need for a user to use their dominant hand is warranted.

Our exploratory study did not consider measures of accuracy related to interaction time, button size, or button design. The low number of inaccuracies in the present study could well be a feature of the design decision to implement button sizing, button iconography, and spacing based within the guidelines identified by the previous work of [i.e. 3, 7, 11, 14] and our own case study observations (see Section 3). Our future work will incorporate tools that measure performance across these interfaces to provide deeper insights into if and how location, layout, design, and size of buttons in media players might contribute to user success while moving.

Presently we have been unable to identify digital library research that addresses interface design for users in motion. The majority of the related work we present sits within wider fields of investigation and does not specifically address interface design considerations which might be prevalent for digital libraries, especially those used by people on the move. We therefore believe that this area that we begin to address in this paper requires continued and broad work given the prevalence of personal digital libraries available today on the now ubiquitous touch screen mobile device.

\section{Conclusion}

Today's users are interacting with personal digital libraries on a diverse range of devices in increasingly more nuanced environments and daily situations. The varied media that digital libraries contain and the ways in which users expect to interact with their media requires constant investigation to ensure that users are afforded the best interactive experience possible with their chosen digital media library. We report here a study 
of user interface interaction with media player interfaces on mobile touch screen devices. Our study provides one of the few insights into use of touchscreen media player interfaces for users in motion, be that the daily commute or a visit to the gym.

Most participants in our study picked up the device with their right hand. While interacting with a test condition, the participants were more likely to use their right hand and right thumb to interact. Considering total interactions, most participants tended to use a single hand when interacting. Given these results we recommend that designers develop mobile digital library interfaces that afford single handed interaction.

Further, vertical layout of media player buttons provided the greatest opportunity for users to use only one hand, while horizontal layout of buttons saw the greatest number of participants needing to switch hands while interacting. When asked users suggested horizontal layout of media player buttons was their preference. Our case study of the current design conventions for media players showed typical media player button placements to be horizontal and therefore we hypothesise the preferences reported are likely due to familiarity. Given our results that suggest improved efficiency with these uncommon button layouts we would recommend further investigation into media player interfaces for users in motion.

Our results and recommendations here pertain to the specific use-case of digital media player interfaces on touch screen mobile devices for users in motion. We argue that these recommendations and the requirement for future work in this area also exists more broadly in the digital library field. Personal digital libraries today encompass a variety of media, be that audio, video, still image, or multi-page text-based documents. Users are likely to interact with these various media on their personal mobile device in a range of individual and shared situations, whether they are sitting, standing, or indeed moving. We, therefore, recommend investigation of the use of digital library interfaces on mobile devices as well as design interventions that support single-handed interaction in ways that may not be the current standard for a given use-case. 


\section{Appendix A}

The six test conditions developed and tested with 30 participants while they walked. TC1-TC3 (the three tested horizontal test conditions) can be seen in the top row, with the vertical test conditions, TC4-TC6 in the bottom row.

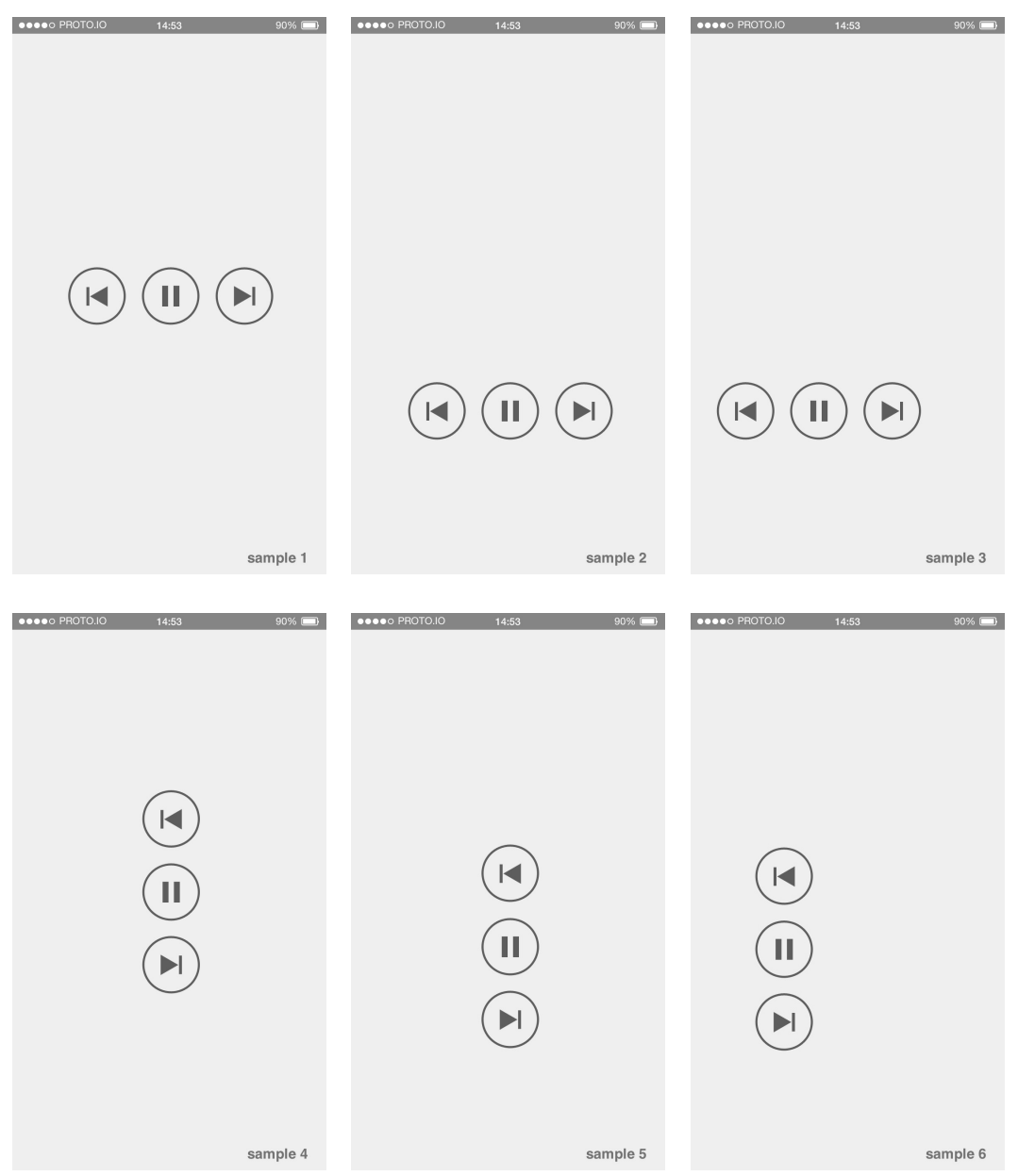




\section{References}

1. Azenkot, S., \& Zhai, S. (2012, September). Touch behavior with different postures on soft smartphone keyboards. In Proceedings of the 14th international conference on Humancomputer interaction with mobile devices and services (pp. 251-260). ACM.

2. Buschek, D., Hackenschmied, M., \& Alt, F. (2017, September). Dynamic UI Adaptations for One-Handed Use of Large Mobile Touchscreen Devices. In IFIP Conference on HumanComputer Interaction (pp. 184-201). Springer, Cham.

3. Conradi, J., Busch, O., \& Alexander, T. (2015). Optimal touch button size for the use of mobile devices while walking. Procedia Manufacturing, 3, 387-394.

4. Cornelia, L. (2014). Do users interact with their mobile devices with their dominant hand? Retreived from http://www. https://realites-paralleles.com/.

5. Chourasia, A. O., Wiegmann, D. A., Chen, K. B., Irwin, C. B., \& Sesto, M. E. (2013). Effect of sitting or standing on touch screen performance and touch characteristics. Human Factors, 55(4), 789-802.

6. Jin, Z. X., Plocher, T., \& Kiff, L. (2007, July). Touch screen user interfaces for older adults: button size and spacing. In International Conference on Universal Access in HumanComputer Interaction (pp. 933-941). Springer, Berlin, Heidelberg.

7. Hall, J. (2012, December 26). MP3 players are dead. Retrieved http://www https://www.businessinsider.com.

8. Hoober, S. (2017, May 8). Design for Fingers, Touch, and People, Part 2. Retrieved from http://www.uxmatters.com

9. Karlson, A. K., Bederson, B. B., \& Contreras-Vidal, J. (2006). Understanding single-handed mobile device interaction. Handbook of research on user interface design and evaluation for mobile technology, 1, 86-101.

10. Parhi, P., Karlson, A. K., \& Bederson, B. B. (2006, September). Target size study for onehanded thumb use on small touchscreen devices. In Proceedings of the 8th conference on Human-computer interaction with mobile devices and services (pp. 203-210). ACM.

11. Park, Y. S., \& Han, S. H. (2010). Touch key design for one-handed thumb interaction with a mobile phone: Effects of touch key size and touch key location. International journal of industrial ergonomics, 40(1), 68-76.

12. Punchoojit, L., \& Hongwarittorrn, N. (2017). Usability studies on mobile user interface design patterns: a systematic literature review. Advances in Human-Computer Interaction, 2017.

13. Sun, X., Plocher, T., \& Qu, W. (2007, July). An empirical study on the smallest comfortable button/icon size on touch screen. In International Conference on Usability and Internationalization (pp. 615-621). Springer, Berlin, Heidelberg.

14. Tao, D., Chen, Q., Yuan, J., Liu, S., Zhang, X., \& Qu, X. (2017, July). Effects of key size, gap and the location of key characters on the usability of touchscreen devices in input tasks. In International Conference on Engineering Psychology and Cognitive Ergonomics (pp. 133-144). Springer, Cham.

15. Trudeau, Matthieu B., Justin G. Young, Devin L. Jindrich, and Jack T. Dennerlein. "Thumb motor performance varies with thumb and wrist posture during single-handed mobile phone use." Journal of biomechanics 45, no. 14 (2012): 2349-2354.

16. Vanderschantz, N., Timpany, C., \& Feng, C. (2018). Tertiary Students' Preferences for Library Search Results Pages on a Mobile Device. In International Conference on Asian Digital Libraries (pp. 213-226). Springer, Cham. 